\title{
Patología orofacial en niños nacidos en condiciones de alto riesgo. Estudio piloto
}

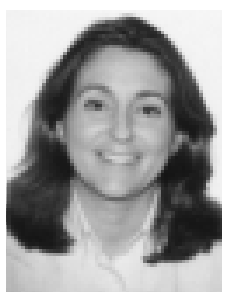

Saavedra-Marbán, Gloria

\section{Orofacial pathology in children born under high- risk conditions. A pilot study}

\section{Saavedra- Marbán, Gloria * Planells del Pozo, Paloma ** Ruiz: Extremera, Ángela ***}

\section{* Doctora en Odontología ** Profesora Titular de Odontopedia- tría. Facultad de Odontología. UCM. *** Profesora Titular de Pediatría. Fa- cultad de Medicina. Universidad de Granada.}

Estudio multidisciplinario, financiado por el Fondo de Investigación Sanitaria (FIS).

\section{Correspondencia}

Gloria Saavedra Marbán

C/ General Pardiñas 103, bajo izda. 28006 Madrid
Resumen: Introducción: los niños nacidos en condiciones de alto riesgo presentan mayor riesgo de padecer enfermedades. A nivel oral observamos en estudios previos hipoplasias de esmalte, caries y alteraciones en paladar, fundamentalmente. Nuestro objetivo fue conocer la patología orofacial y su relación con el bajo peso al nacimiento en una población de niños nacidos en condiciones de alto riesgo de Granada. Pacientes y método: la muestra fue de 60 niños de 46 años nacidos en condiciones de alto riesgo y que necesitaron cuidados intensivos neonatales. Se realizó un estudio descriptivo para determinar la patología en estos niños y un análisis estadístico univariante para analizar las variables según el peso en el nacimiento. Resultados: observamos una relación estadísticamente significativa entre el peso al nacimiento menor de 1550 gramos y las variables perinatales. El porcentaje de anomalías estructurales dentarias, caries, hábitos orofaciales y traumatismos observado en nuestra muestra fue elevado, existiendo relación estadísticamente significativa con el peso al nacer. Los hábitos higiénicos y asistencia dental previa fueron escasos, no estando relacionados con el peso en el nacimiento. Conclusiones: es importante considerar los datos neonatales ya que aportan información sobre la presencia de secuelas en el paciente infantil nacido en condiciones de riesgo y permitirá al odontopediatra establecer protocolos preventivos y de atención al niño.

Palabras clave: Niños, Alto riesgo, Oral, Patología.

Abstract: Introduction: Children born in high-risk conditions present a higher risk of suffering from illnesses. At the oral level, previous studies have mainly observed enamel hypoplasia, dental caries and palate anomalies. Our objective was to know orofacial pathology and its relation with low birth weight in a group of children born in Granada, Spain, under high-risk conditions. Patients and method: The sample comprised 60 children between 4-6 years born in high-risk conditions who needed neonatal intensive care. A descriptive study was carried out to determine the pathology in these children as well as a single variant statistical analysis to analyse the variables in terms of birth weight. Results: We observed a statistically significant relationship between birth weight under 1,550 grams and perinatal variables. The percentage of dental structure anomalies, dental caries, orofacial habits and traumatised teeth observed in our sample was high, showing a statistically significant relationship with birth weight. The hygienic habits and previous dental assistance were scarce, not being related with birth weight. Conclusions: The neonatal data are important and have to be taken into account. They supply information about sequels in children born in high-risk conditions and will allow the paediatric dentist to establish preventive and therapeutic protocols for the children.

Key words: Children, High-risk, Oral, Pathology.

\begin{tabular}{ccc}
\hline Fecha recepción & Fecha última revisión & Fecha aceptación \\
$10-09-2003$ & $17-03-2004$ & $30-03-2004$ \\
\hline
\end{tabular}

BIBபD [1138-123X (2004)9:2; marzo-abril 125-256]

Saavedra-Marbán G, Planells del Pozo P, Ruiz-Extremera A. Patología orofacial en niños nacidos en condiciones de alto riesgo. RCOE 2004;9(2):151-158. 


\section{Introducción}

En medicina, particularmente en pediatría, los avances científicos han dado como resultado la disminución de la mortalidad perinatal e infantil. Los factores que han contribuido a ello son múltiples, pero sin duda el avance en la aplicación de la terapia intensiva pediátrica con tecnología cada vez más avanzada, en las Unidades de Cuidados Intensivos Neonatales, junto con la detección e intervención en los embarazos de alto riesgo, constituyen los pilares que fundamentan estos datos. Sin embargo, existen datos que señalan la necesidad de seguimiento de los recién nacidos de alto riesgo, ya que en muchas ocasiones la disminución de la mortalidad puede llevar aparejado un incremento importante de la morbilidad. Ciertos investigadores concluyen que el número de niños con trastornos discapacitantes crónicos casi se ha triplicado en los últimos treinta años en todos los países desarrollados y sus tasas de supervivencia han aumentado de tal manera que el $90 \%$ de ellos alcanza la edad adulta.

Conceptualmente, los niños nacidos en condiciones de alto riesgo biológico son definidos por los investigadores $s^{1-5,6^{*+7 * *}}$ como aquellos que presentan problemas que ponen en peligro su salud durante el periodo prenatal, en el momento del nacimiento o en la etapa postnatal. Dentro de los niños considerados de alto riesgo, los niños prematuros o con bajo peso en el momento del nacimiento constituyen el grupo más numeroso.

Hoy en día, debido a los importantes avances técnicos, observamos una disminución de la mortalidad de pre- maturos extremadamente pequeños (menores de 1000-1500 gramos) y un gran aumento de la morbilidad (prematuros nacidos con bajo peso que sobreviven con secuelas). Los estudios publicados sobre el seguimiento de estos niños prueban que constituyen un grupo con peligro de padecer más enfermedades ${ }^{1,2}$ y los niños de más bajo peso al nacimiento son los que más secuelas van a presentar ${ }^{3,4}$. Por lo tanto, será muy importante evaluar periódicamente al paciente para normalizar lo más pronto posible su situación ${ }^{5}$. Con esta intención, hace años se empezaron a crear Unidades de Seguimiento y Atención Temprana para intervenir en los problemas detectados y para poder evaluar periódicamente los resultados obtenidos de la asistencia neonatal $\mathrm{l}^{6 * * 7 * * *}$.

Todo el seguimiento y tratamiento en estos niños se debería realizar mediante un equipo multidisciplinario, donde se incluirían obstetras, neonatólogos, neuropediatras, psicólogos, fisioterapéutas, pediatras de atención primaria, personal de enfermería, logopedas, oftalmólogos, otorrinolaringólogos y odontopediatras, entre otros. Pero la realidad es que en pocos centros se incluye al odontopediatra como miembro de este equipo multidisciplinario y uno de los problemas comunes a muchos de ellos y menos atendido es el estado de salud oral; las grandes necesidades médicas pueden haber motivado que el área oral haya sido poco atendida en estos pacientes en sus revisiones. Una vez resueltos los problemas vitales prioritarios, deberemos informar y educar a los padres sobre la salud oral de sus hijos e instaurar programas de prevención, ya que estos niños, debido a sus es- peciales condiciones perinatales, siempre serán considerados como pacientes de riesgo.

Según la literatura previa, los niños prematuros de bajo peso presentan una mayor cantidad de patología estructural del esmalte y la dentina, diferencias en la cronología de erupción, caries, alteraciones en el paladar....$^{* * 9,10^{*}-14}$. Hasta el momento no existen estudios en nuestro país para conocer si en nuestro ámbito existe también esta patología y así poder determinar las necesidades dentro del equipo multidisciplinario. Además muchos de estos niños llegarán a las consultas dentales requiriendo cuidados, por lo que es importante conocer el alcance de sus secuelas ya que estas alteraciones pueden ser patentes aún en la adolescencia ${ }^{3}$.

\section{Objetivos}

1. Conocer el estado de salud oral en una población de niños nacidos en condiciones de alto riesgo.

2. Conocer la relación entre las variables orales y el bajo peso al nacimiento en dicha población.

\section{Pacientes y método}

Este estudio se ha llevado a cabo en el Hospital Clínico Universitario "San Cecilio" de Granada (España), en el Departamento de Pediatría y más concretamente en la Unidad de Seguimiento y Estimulación precoz, durante los años 1997-00.

Esta investigación forma parte de un estudio multidisciplinario, financiado por el Fondo de Investigación Sani- 
tario (FIS). En este estudio participaron un gran número de profesionales (obstetras, neonatólogos, psicólogos, fisioterapéutas, personal de enfermería, neuropediatras, logopedas...) al que se incorporó la figura del odontopediatra.

\section{Selección de la muestra}

Los criterios de inclusión y exclusión fueron los siguientes:

\section{Criterios de inclusión}

- Niños de 4-6 años de edad en el momento de la exploración.

- Niños nacidos en el Hospital Clínico San Cecilio de Granada en condiciones de alto riesgo y sometidos a hospitalización en la Unidad de Cuidados Intensivos Neonatales del citado centro sanitario.

\section{Criterios de exclusión}

- Niños portadores de alguna cromosomopatía.

- Niños con dismorfia cráneo-facial de origen genético.

El total de pacientes fue de 60 niños escogidos al azar entre un grupo de niños nacidos en condiciones de alto riesgo y que necesitaron ser ingresados en la UCIP (Unidad de Cuidados Intensivos Pediátricos) tras el nacimiento, causado por parto prematuro y bajo peso al nacer, o por enfermedades en el postparto que hicieron imprescindible su ingreso en $\mathrm{UCl}$.

Se informó a los padres que en las revisiones generales de estos niños se incluiría una revisión oral para valorar la patología existente a este nivel. Todos los niños al comienzo del estudio debían poseer el correspondiente consentimiento informado por parte de los padres o tutores.

En una primera fase del estudio se recogieron los expedientes clínicos de cada niño y se elaboró una ficha individualizada de cada paciente donde figuraban los datos relativos al nacimiento de los niños y datos de la madre en estado de gestación, aportados por los neonatólogos. Se recogieron 11 datos referentes a antecedentes neonatales: edad gestacional, peso al nacer, talla al nacimiento, días en UCIP, días de hospitalización, ventilación mecánica, tiempo que fue necesaria la ventilación mecánica, edad de la madre, transfusiones, retinopatía y valoración obtenida con test psicológicos de los niños. Se recogió igualmente cualquier patología que apareciera durante la hospitalización y secuelas de aparición tardía que fueron diagnosticadas durante las revisiones periódicas.

Paralelamente se realizaron entrevistas a los padres o tutores de antecedentes médicos e historia médica actual y de aspectos relativos a la salud oral (52 items) a fin de conocer y analizar factores como antecedentes de patología oral, cuidados higiénicos habituales y antecedentes de hábitos y traumatismos entre otros.

Tras la recogida de los datos, se realizó una exploración bucal, por un único investigador, odontopediatra, según directrices de la OMS. En la exploración se observaron: aspectos preventivos como índice de placa dental y presencia de selladores, erupción, características de la oclusión, anomalías dentarias, anomalías maxilofaciales, donde se observaron fundamentalmente consecuencias de hábitos y problemas en el paladar y el estado dentario: caries y restauraciones presentes en la boca. Tras la exploración se realizaron fotografías con la finalidad de recoger registros objetivos de cada caso.
Posteriormente se tomaron impresiones de alginato de ambas arcadas y una cera de oclusión para la confección de modelos de estudio.

\section{Metodología de análisis de los datos}

Para el estudio descriptivo, las variables cuantitativas fueron analizadas como media aritmética y desviación estándar de la media y por distribución en percentiles. Las variables cualitativas se expresaron como número de pacientes y porcentajes.

Tras el análisis descriptivo se realizó un estudio estadístico univariante para comprobar si existía asociación estadísticamente significativa entre cada variable y el bajo peso al nacimiento. En este análisis, las variables cuantitativas se analizaron mediante el método de Bonferroni y Neuman-Keuls de comparaciones múltiples y las cualitativas mediante el test de la Chi cuadrado o la prueba exacta de Fisher. En todos los test e intervalos de confianza se consideró un nivel de significación estadística del 95\%

\section{Resultados}

\section{Estudio descriptivo}

\section{Aspectos demográficos y antece- dentes perinatales}

Los datos demográficos de la muestra (edad de la madre en el momento del parto, sexo, edad gestacional, peso al nacimiento y talla) quedan reflejados en la tabla 1.

El 53\%de los niños precisaron ventilación mecánica. El valor medio del tiempo de ventilación mecánica, de los días en UCIN y los días de hospita- 


\begin{tabular}{|c|c|c|c|c|c|}
\hline \multicolumn{6}{|c|}{ Tabla l: Datos demográficos de la muestra estudiada } \\
\hline Variable & Media & $\begin{array}{l}\text { Desviación } \\
\text { estándar }\end{array}$ & $\begin{array}{l}\text { Error } \\
\text { stándar } \\
\text { media }\end{array}$ & $\begin{array}{l}\text { Valor } \\
\text { mínimo }\end{array}$ & $\begin{array}{l}\text { Valor } \\
\text { máximo }\end{array}$ \\
\hline Edad madre (años) & 25,767 & 5,5613 & 0,71796 & 17 & 38 \\
\hline Edad gestacional (semanas) & 32,383 & 4,923 & 0,55413 & 24 & 43 \\
\hline Peso al nacer $(\mathrm{g})$ & 1857 & 866,75 & 111,90 & 720 & 4100 \\
\hline Talla $(\mathrm{cm})$ & 42,067 & 5,9883 & 0,77309 & 30 & 56 \\
\hline
\end{tabular}

\begin{tabular}{|c|c|c|c|c|c|}
\hline \multicolumn{6}{|c|}{ Tabla 2: Antecedentes neonatales de la muestra estudiada } \\
\hline Variable & Media & $\begin{array}{l}\text { Desviación } \\
\text { estándar }\end{array}$ & $\begin{array}{c}\text { Error } \\
\text { stándar } \\
\text { media }\end{array}$ & $\begin{array}{l}\text { Valor } \\
\text { mínimo }\end{array}$ & $\begin{array}{c}\text { Valor } \\
\text { máximo }\end{array}$ \\
\hline Días en UCIN & 25,362 & 27,907 & 3,6644 & 0 & 100 \\
\hline Días hospitalización & 50,362 & 36,628 & 4,8095 & 0 & 150 \\
\hline Días VM & 12,559 & 14,209 & 2,4368 & 1 & 53 \\
\hline
\end{tabular}

lización que precisaron los niños queda reflejado en la tabla 2.

\section{Datos de salud oral recogidos en la encuesta}

En cuanto a los datos de salud oral, se les preguntó por la asistencia al dentista. Destaca que el $62 \%$ no había asistido nunca a una consulta odontológica.

En cuanto a la frecuencia del cepillado, un $21,7 \%$ de la muestra no se cepilló nunca o muy esporádicamente, un $75 \%$ se cepillaron 1 o 2 veces al día y un $3 \%$ de los niños, 3 o más veces al día.

Acerca de los hábitos, el $67 \%$ de los niños presentaba algún tipo indeseable de hábito. El 34\%de los niños con hábitos, presentó respiración oral. Del total de niños de la muestra, un 15\%presentó deglución atípica, un $8 \%$ succión digital, un $17 \%$ hábito de chupete y un $10 \%$ de chupe- teo de diferentes objetos. Otros hábitos recogidos fueron la onicofagia, presentado en un $17 \%$ de la muestra y el bruxismo, que observamos en el $13 \%$

En los resultados relativos a los antecedentes de traumatismos, se obtuvo que un $45 \%$ de los niños había sufrido algún traumatismo dentario.

\section{Datos de salud oral recogidos en la exploración intraoral}

En un $78 \%$ de los pacientes se encontró un índice de placa muy elevado. Ninguno de los niños explorados presentaron selladores de fosas y fisuras en sus molares.

En cuanto a la existencia de caries, un $55 \%$ de los niños no presentó ninguna caries y en un $6,6 \%$ del total de los niños explorados, se observó la existencia de algún tratamiento restaurador.
Las anomalías observadas más frecuentemente fueron las de estructura, advertidas en un $47 \%$ de niños. De entre ellos, un 7\%tenían un solo diente afectado, un 32\% 2-4 dientes con alteración, un 29\%4 a 6 dientes dañados y un $32 \%$ anomalías estructurales en más de seis dientes.

\section{Análisis de las variables según el peso al nacer}

Antecedentes perinatales de los pacientes según el peso al nacimiento

En la tabla 3 se observan las características de los pacientes según su peso al nacer. El percentil $50(P-50)$ del peso al nacimiento correspondió a 1550 g. La valoración de la edad gestacional, talla al nacimiento y las diferentes intervenciones terapéuticas mostraron diferencias muy significativas.

\section{Relación del peso al nacimiento con} los datos de la salud oral recogidos en la encuesta

En la tabla 4 observamos que no se ha encontrado ninguna diferencia significativa en la asistencia al dentista, el cepillado dental, la presencia de placa bacteriana, uso de fluoruros y presencia de selladores entre ambos grupos.

Relación del peso al nacimiento con los datos de salud oral recogidos en la exploración intraoral

En cuanto a los factores observados en la exploración intraoral no se apreciaron diferencias significativas entre ambos grupos en cuanto a la presencia de anomalías estructurales, caries y traumatismos, evaluados globalmente.

Se obtuvo una diferencia cercana a la significación $(p=0,06)$ en el número 


\begin{tabular}{|c|c|c|c|}
\hline & $\begin{aligned} \text { Peso } & \leq 1550 \mathrm{~g} \\
\mathbf{N} & =\mathbf{3 1}\end{aligned}$ & $\begin{array}{c}\text { Peso }>1550 \mathrm{~g} \\
\mathrm{~N}=\mathbf{2 9}\end{array}$ & $\mathbf{P}$ \\
\hline Edad madre $\mu \pm \mathrm{DS}$ & $25,5 \pm 5$ & $26,4 \pm 6$ & NS \\
\hline Parto por cesárea n (\%) & $19(61)$ & $19(65)$ & NS \\
\hline Sexo masculino $n(\%)$ & $19(63)$ & $23(79)$ & NS \\
\hline Edad Gestacional $\mu \pm \mathrm{DS}$ & $30,5 \pm 2.7$ & $35,6 \pm 4$ & 0,0001 \\
\hline Talla al nacimiento $\mu \pm \mathrm{DS}$ & $37,6 \pm 4$ & $47 \pm 5$ & 0,001 \\
\hline Ventilación Mecánica n (\%) & $25(81)$ & $8(28)$ & 0,0001 \\
\hline Días de VM $\mu \pm$ DS & $16 \pm 15$ & $4 \pm 3$ & 0,01 \\
\hline Días Estancia en UCIN $\mu \pm$ DS & $46 \pm 29$ & $9 \pm 7$ & 0,001 \\
\hline Días Estancia en Hospital $\mu \pm$ DS & $79 \pm 29$ & $23 \pm 14$ & 0,001 \\
\hline \multicolumn{4}{|c|}{$\begin{array}{l}\text { Nota: (u): Media aritmética, (DS): Desviación estándar, (n): Número de niños, (VN } \\
\text { Ventilación Mecánica, (UCIN): Unidad de Cuidados Intensivos Neonatales. }\end{array}$} \\
\hline
\end{tabular}

\begin{tabular}{|c|c|c|c|c|c|}
\hline \multicolumn{6}{|c|}{$\begin{array}{c}\text { Tabla 4: Caracteristicas de los pacientes según } \\
\text { peso al nacimiento y salud oral }\end{array}$} \\
\hline & $\begin{array}{c}\text { Total } \\
\mathbf{N}=60\end{array}$ & IC al 95\% & $\begin{array}{l}\text { Peso } \\
\leq 550 \mathrm{~g} \\
\mathrm{~N}=31 \\
\end{array}$ & $\begin{array}{l}\text { Peso } \\
>1550 \mathrm{~g} \\
\mathrm{~N}=29 \\
\end{array}$ & $\mathbf{P}$ \\
\hline $\begin{array}{l}\text { Asistencia al } \\
\text { odontólogo n (\%) }\end{array}$ & $21(35)$ & $23.5 \% 48.5 \%$ & $12(39)$ & $9(31)$ & NS \\
\hline $\begin{array}{l}\text { Cepillado de } \\
\text { dientes } n(\%)\end{array}$ & $46(77)$ & $64 \% 86 \%$ & $25(81)$ & $21(72)$ & NS \\
\hline Placa n (\%) & $51(85)$ & $73 \% 92 \%$ & $26(84)$ & $25(86)$ & NS \\
\hline Uso de Flúor n (\%) & $4(7)$ & $2 \% 17 \%$ & $1(3)$ & $3(10)$ & NS \\
\hline Selladores n (\%) & $0(0)$ & & $0(0)$ & $0(0)$ & NS \\
\hline Nota: $(\mathrm{n})$ : Número de $\mathrm{n}$ & & & & & \\
\hline
\end{tabular}

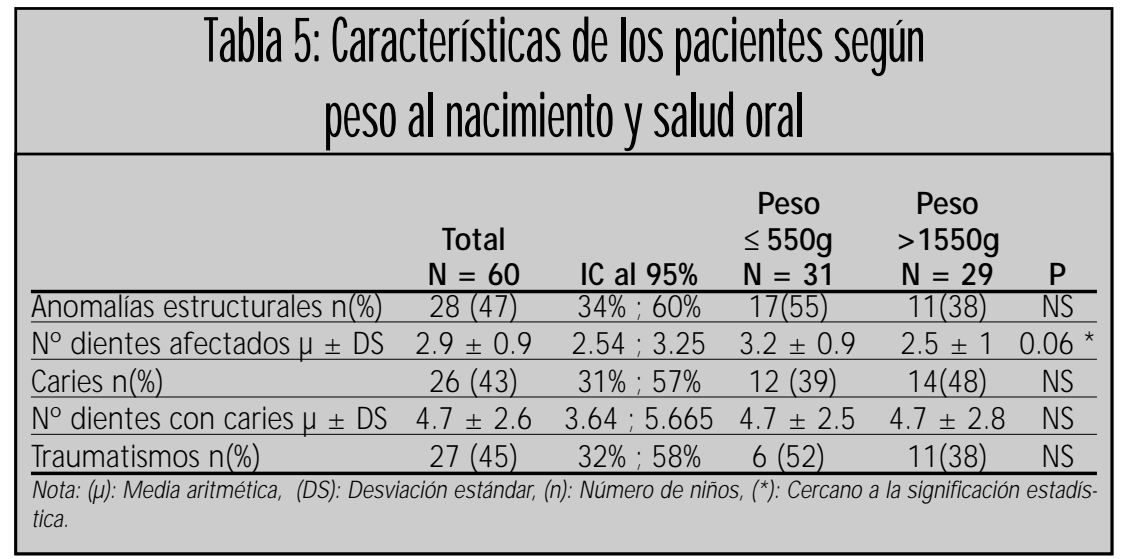

de dientes afectados por anomalías estructurales observándose que los niños con menor peso al nacimiento tenían un mayor número de dientes dañados (tabla 5).

Respecto a la presencia de algún hábito, no se encontraron diferencias significativas entre ambos grupos, aunque observamos que los niños de menor peso tenían una tendencia mayor a presentarlos. Al observar cada tipo de hábito, mostraron diferencias significativas según el peso al nacimiento los de chupeteo de objetos, con un $\mathrm{p}=0,01$.

\section{Discusión}

Las investigaciones realizadas sobre niños nacidos en condiciones de alto riesgo (bajo peso y prematuridad) son numerosas en cuanto a temas pediátricos, pero las investigaciones que tratan de aspectos odontológicos son muy escasas. Esto puede ser debido a que hace años había una elevada mortalidad de estos niños y eran los aspectos médicos relativos a tasas de supervivencia, los que ocupaban más a los investigadores; actualmente, dado que la esperanza de vida ha aumentado, es muy importante avanzar en el conocimiento de las secuelas orofaciales, ya que es el mejor camino hacia su prevención.

Los aspectos orofaciales más estudiados a nivel internacional son los defectos estructurales de los tejidos dentarios, la erupción y maduración dentaria y la patología cariosa. Además de estos parámetros estudiados con anterioridad en la literatura, hemos incluido en el presente estudio, el análisis de los traumatismos y la 
presencia de hábitos orofaciales. Pensamos que podía ser interesante debido a los antecedentes médicos en forma de secuelas con problemas de discapacidad sensorial, motora y psicológica que suelen presentar estos pacientes.

Otros parámetros que hemos analizado en nuestro estudio y que igualmente no hemos podido encontrar en otros análisis anteriores, son los datos referidos a antecedentes odontológicos como asistencia al dentista, medidas preventivas y hábitos higiénicos entre otros.

\section{Defectos estructurales de los tejidos duros dentarios}

En nuestro estudio hemos encontrado un $47 \%$ de niños con defectos estructurales del esmalte. Los porcentajes obtenidos por los distintos autores van desde un $21 \%{ }^{14}$ a un $100 \% \%^{1,16}$. Todos los autores afirman que existe un mayor número de defectos estructurales dentarios en niños nacidos prematuramente, de bajo peso o con enfermedades en el periodo neonatal, pero en lo que no existe un acuerdo entre los investigadores es en la etiología de estos defectos. Los citados autores piensan que pueden deberse a factores generales, locales o ambos de forma conjunta.

Los factores generales que observan los investigadores, posibles causantes de los defectos, son el bajo peso, prematuridad o enfermedades en periodo neonatal ${ }^{9,10 *, 14-19}$. En todos estos estudios se advierte una característica común y es la aparición de los defectos de forma simétrica en la dentición.

Problemas en el periodo neonatal que se han asociado a la patogé- nesis de la hipoplasia han sido asfixia neonatal, síndrome de distress respiratorio, rickets neonatal (severo disturbio del calcio), desórdenes nutricionales como hipocalcemia, preeclampsia materna, osteopenia y diabetes ${ }^{9,19-23}$. Las infecciones son otro factor general estudiado, ya que los niños prematuros que nacen con bajo peso tienen más riesgo de padecer infecciones al nacer, pues su sistema inmunológico es más débil; esto hace que por una infección pueda alterarse la formación de los tejidos duros del diente y producirse lesiones de tipo hipoplasia.

En los niños con rickets neonatal, Seow y cols $^{8 * *}$ y Nikiforuk y Fraser ${ }^{15}$ encontraron un $100 \%$ de defectos, ya que son niños con un severo disturbio del metabolismo del calcio que podría ser el responsable de estos defectos. Johnsen y cols en un estudio realizado en $1984^{13}$, muestra la importancia del bajo peso sin ningún otro factor sistémico en la patogénesis de la hipoplasia.

Existe otro grupo de autores que piensan que son los factores locales, como el uso del laringoscopio e intubación endotraqueal usado para la ventilación mecánica, los causantes de los defectos dentarios ${ }^{10^{*}, 24}$. Estos autores observaron más defectos en el lado donde se aplica el laringoscopio que en el contralateral.

Seow y cols ${ }^{9}$, en otro estudio realizado en 1984, observó los defectos estructurales que tenían los niños prematuros y encontró una relación significativa entre la presencia anomalías y los pacientes intubados por necesidad de ventilación mecánica (85\% frente a $21,7 \%$ ).

Kopra y Davis ${ }^{24}$ observaron que de los niños prematuros de bajo peso que necesitaron intubación, el 23-29\% tenían defectos de esmalte, mientras que el resto de prematuros de bajo peso que no precisaron intubación, carecía de dicho defecto.

Otro grupo de autores ${ }^{9,12,25}$ se inclinan por una mezcla de ambos factores, locales y generales. Para otros investigadores, los datos estadísticamente significativos se establecen entre los defectos estructurales y la necesidad de ventilación mecánica e igualmente entre los defectos y haber padecido enfermedades en el período neonatal ${ }^{12}$.

En nuestro estudio los niños que recibieron ventilación mecánica, lo hicieron por vía nasal, por lo que los defectos que observamos podrían deberse en un principio a causas generales como el parto prematuro, peso bajo al nacimiento, infecciones y problemas neonatales, o a la deprivación de oxígeno de los ameloblastos durante un período determinado, pero no como señalan otros investigadores, por el decúbito de la ventilación mecánica en la cavidad oral. Ahora bien, las maniobras de intubación mecánica son frecuentes durante el periodo de estancia en las Unidades de Cuidados Intensivos Pediátricos y, en muchas ocasiones, estas se realizan en condiciones de urgencia vital para el niño, con lo cual puede ejercerse cierto grado de presión o trauma sobre los rodetes alveolares en el niño, que pueden justificar la aparición de anomalías dentarias en forma de secuelas en su estructura.

\section{Patología cariosa}

Los pacientes prematuros de bajo peso son considerados, por la mayoría 
de los investigadores, como pacientes con alto riesgo de caries.

Se cree que hay más predisposición a la caries en los dientes con defectos estructurales, pero la importancia de los defectos de esmalte frente a otros factores de riesgo de caries como higiene, flujo salival, alimentación o infecciones es desconocida ${ }^{14,16,25}$.

Hay contradicciones entre los distintos autores en cuanto a que en los niños prematuros de bajo peso hay mayor prevalencia de caries que en los niños nacidos a término. Los que opinan que hay mayor prevalencia creen que se debe a que estos niños tienen una mayor presencia de dientes con hipoplasia $u$ otros defectos que son más susceptibles a la caries, defectos del tipo «hipoplasia con opacidad $»^{14,19,26}$. Otros investigadores opinan que no hay relación entre los defectos de la estructura y la caries dental, observando en su estudio menor número de caries que en el grupo control ${ }^{4}$. Concluyen que estos datos son debidos a que los niños prematuros que componen su muestra están muy vigilados y motivados para la higiene oral ${ }^{12,14}$.

En la presente investigación, hemos obtenido un $55 \%$ de niños sin ninguna caries, no observando mayor presencia de éstas en los dientes con hipoplasia.

A los doctores y personal de la Unidad de Seguimiento y Estimulación Precoz del Hospital Clínico San Cecilio de Granada, por la ayuda prestada pa-

\section{Problemas en la madura- ción dentaria y cronología de erupción.}

Los problemas perinatales tienen un fuerte impacto sobre el desarrollo dentario ${ }^{27}$. Según los estudios, el retraso en la formación y erupción dentaria se observa en estos pacientes hasta los veinticuatro meses de vida y se debe al nacimiento prematuro. Mediante el manejo de las edades cronológicas y las edades corregidas se puede explicar el retraso ${ }^{11,28}$. En los estudios se observa que cuanto menor es el peso al nacimiento, menor la edad gestacional y más problemas perinatales, el retraso en la maduración y erupción dentaria es mayor ${ }^{29}$.

En estudios como el nuestro, con edades en los pacientes entre los $4 \mathrm{y}$ 6 años, no observamos retraso en la erupción, aunque es posible que estos niños hubieran presentado un retraso en la formación y erupción dentaria hasta los dos años de edad ${ }^{30}$.

\section{Conclusiones}

1. Hemos observado una elevada patología oral (anomalías estructurales de los tejidos duros dentarios, caries, hábitos orofaciales, traumatismos) en los niños nacidos en condiciones de alto riesgo.

2. Existe relación estadísticamente significativa entre anomalías estructu-

\section{Agradecimientos}

ra poder realizar la exploración y obtención de los registros de los niños.

A las personas que han colaborado en la obtención de registros. rales, respiración oral y succión no nutritiva y el menor peso al nacer.

3. Es importante valorar en la historia clínica y anamnesis los datos referentes al periodo neonatal, pues nos pueden ofrecer importante información sobre la patología oral presente en los pacientes infantiles nacidos en condiciones de riesgo.

4. Los protocolos de prevención, atención y seguimiento odontopediátrico en estos niños, deben ser incluidos dentro de sus revisiones periódicas en los programas de atención temprana. Es fundamental que el odontopediatra forme parte del equipo multidisciplinar que se ocupa del seguimiento de estos niños.

5. Se necesita un mayor número de estudios en nuestro país, para acercarnos a la posible etiología de estos problemas.

\section{Nuevas directrices}

De los resultados de la investigación precedente se crea una línea de investigación que, nuevamente financiada por el Fondo de Investigación Sanitaria, contempla entre sus objetivos la valoración mineral de los tejidos duros dentarios procedentes de los niños de la muestra, así como la valoración de las secuelas maxilofaciales evidenciadas en esta población infantil.

A los niños y padres que han participado en el estudio, por su colaboración, sin la cual no hubiera sido posible la realización de este trabajo. 


\section{Bibliografía recomendada}

Para profundizar en la lectura de este tema, el/los autor/es considera/an interesantes los artículos que aparecen señalados del siguiente modo: *de interés $* *$ de especial interés.

1. Perlman M, Claris O, Hao Y, y cols. Secular changes in the outcomes to eighteen to twenty-four months of age of extremely low birth weight infants, with adjustment for changes in risk factors and severity of illness. J Pediatr 1995;126:75-87.

2. Cohen S. Longitudinal studies of preterm infants. En: Taeush $\mathrm{H}$ and Yogman $\mathrm{M}$, editores. Follow-up management of the high-risk infant. Boston/Toronto: Little, Brown and Company, 1987:21-8

3. Waldman HB. Low birth-weight and the relationship to developmental problems. J Dent Child 1996;63:354-7.

4. Arrabal-Terán C, Arizcun-Pineda J. El niño que fue prematuro: cuidados. En: De Paz Garnelo JA Pediatría Preventiva y Social. Madrid: Interbindersal, 1997:77-85

5. Arrabal-Terán C, Arizcun-Pineda J. Recién nacido de bajo peso: cuidados y transporte. En: De Paz Garnelo JA Pediatría Preventiva y Social. Madrid:s Interbindersal, 1997:67-75

6**. Ruiz Extremera A, Robles Vizcaíno C. Prevención, atención y seguimiento de niños en riesgo o con lesiones establecidas. Granada: Editorial Comares, 1997.

Este texto en general nos da una visión muy importante desde el punto de vista de las distintas especialidades hospitalarias de las lesiones que pueden tener los niños que nacen en condiciones de alto riesgo y por lo tanto como hacer su prevención y seguimiento.

7**. Ruiz Extremera A, Robles Vizcaíno C. Niños de riesgo. Programas de Atención temprana. Madrid: Ediciones Norma-Capitel, 2004.

El presente texto refiere con una visión actualizada y multidisciplinar los objetivos de los equipos de responsabilidad de seguimiento en niños nacidos en condiciones de riesgo.

8**. Seow WK, Brown JP, Tudehope D, O'Callaghan M. Dental defects in the deciduous dentition of premature infants with low birth weight and neonatal rickets. Pediatric Dentistry $1984 ; 6: 88-92$

Este artículo nos demuestra como los defectos dentales de estructura (hipoplasia e hipocalcificación) pueden tener causas generales como el bajo peso o severos disturbios del metabolismo del calcio, como el rickets neonatal, en prema- turos. Los autores obtuvieron unos porcentajes de patología muy elevados.

9. Seow WK, Masel JP, Weir C, Tudehope D. Mineral deficiency in the pathogenesis of enamel hypoplasia in prematurely born, very low birthweight children. Pediatric Dentistry 1989;11:297-302.

10*. Seow WK, Brown JP, Tudehope D, O'Callaghan M. Developmental defects in the primary dentition of low birth-weight infants: adverse effects of laryngoscopy and prolonged endotracheal intubation. Pediatric Dentistry 1984; 6:28-31.

Los autores en este artículo nos evidencian como por una causa local, como es el decúbito producido por el laringoscopio en niños que nacidos en condiciones de alto riesgo necesitaron respiración asistida, se producen defectos estructurales dentarios.

11. Seow WK, Humphrys C, Mahanonda R, Tudehope D. Dental eruption in low birth-weight prematurely born children: a controlled study. Pediatric Dentistry 1988;10:39-42.

12. Fearne JM, Bryan E, Brook AH. Enamel defects in the primary dentition of children born weighing less than 2000g. Br Dent $\mathrm{J}$ 1990;168:433-7.

13. Johnsen D, Krejct C, Hack M, Fanaroff A. Distribution of enamel defects and the association with respiratory distress in very low birhtweight infants. J Dent Res 1984;63:59-64.

14. Lai PY, Seow WK, Tudehope D, Rogers Y. Enamel hypoplasia and dental caries in very-low birthweight children: a case-controlled, longitudinal study. Pediatric Dentistry 1997; 19:42-9

15. Nikiforuk G, Fraser D. Chemical determinants of enamel hypoplasia in children with disorders of calcium and phosphate homeostasis. J Dent Res 1979;58:1014-5.

16. Pascoe L, Seow WK. Enamel hypoplasia and dental caries in Australian Aboriginal children: prevalence and correlation between the two diseases. Pediatric Dentistry 1994; 16:193-9.

17. Seow WK. A study of the development of the permanent dentition in very low birthweight children. Pediatric Dentistry 1996;18:379-84.

18. Drummond BK, Ryan S, O'Sullivan E, Congdon P, Curzon M. Enamel defects of the pri- mary dentition and osteopenia of prematurity. Pediatric Dentistry 1992;14:119-21.

19. Norén JG. Enamel structure in deciduous teeth from low-birth-weight infants. Acta Odontol Scand 1983;41:355-62.

20. Larmas M, Hietala E, Simila S, Pajari U. Oral manifestations of familial hypophosphatemic rickets after phosphate supplement therapy: a review of the literature and report of case. J Dent Child 1991;58:328-34.

21. Limeback H, Schlumbohm C, Sen A, Nikiforuk G. The effects of hypocalcemia/ hypophosphatemia on porcine bone and dental hard tissues in an inherited form of type 1 pseudo-vitamin D deficiency rickets. J Dent Res 1992; 71:346-52.

22. Seeto E, Seow WK. Scanning electron microscopic analysis of dentin in vitamin D-resistant rickets- assessment of mineralization and correlation with clinic findings. Pediatric Dent 1991;13:43-8.

23. Goodman J, Gelbier M, Bennett J, Winter G. Dental problems associated with hypohosphataemic vitamin $\mathrm{D}$ resistant rickets. Int $\mathrm{J} \mathrm{Pa}$ ed Dentistry 1998;8:19-28.

24. Kopra DE, Davis EL. Prevalence of oral defects among neonatally intubated 3- to 5- and 7- to 10-year-old children. Pediatric Dentistry 1991;13:349-55.

25. Seow WK. Enamel hypoplasia in the primary dentition: a review. J Dent Child 1991;58:441-9.

26. Nikiforuk G, Fraser D. The etiology of ename hypoplasia: a unifying concept. J Paediatric Dent 1981;98:888-93.

27.Harris E, Barcroft B, Haydar S, Haydar B. Delayed tooth formation in low birthweight African-American children. Pediatric Dentistry 1993;15:30-5.

28. Fadavi S, Punwani I, Adeni S, Vidyasagar D. Eruption pattern in the primary dentition of premature low-birth-weight children. J Dent Child 1992;59:120-2.

29. Seow WK. A study of the development of the permanent dentition in very low birthweight children. Pediatric Dentistry 1996;18:379-84.

30. Seow WK, Needleman HL, Holm I. Effects of familial hypophosphatemic rickets on dental development: a controled, longitudinal study. Pediatric Dentistry 1995;17:346-50. 